research to practice American. Journal of Clinical Nutrition 2004; 79: 17-21.

6. Kramer MS. Determinants of low birth weight: methodological assessment and meta-analysis. Bulletin of the World Health Organization 1987; 65: 663-737.

7. Nanayakkara KK, Samarakoon AB, Perera BH, et al. Size of newborns at birth. Journal of Obstetrics and Gynaecology Research 2011; 31: 311-4.

8. Annual Health Statistics Sri Lanka 2007. Medical Statistics Unit, Department of Health, Ministry of Health and Nutrition of Sri Lanka. 2008. P54.

9. Freeman MG, Graves WL. Risk of premature delivery among indigent Negro women based on past reproductive performance. Obstetrics and Gynaecology 1969; 34: 648-54.

10. Kaltreider DF, Johnson JWC. Patients at high risk for lowbirth-weight delivery. American Journal of Obstetrics and Gynecology 1976; 124: 251-6.

11. Tejani NA. Recurrence of intrauterine growth retardation. Obstetrics and Gynecology 1982; 59: 329-31.

12. Goldenberg RL, Hoffman HJ, Cliver SP, Cutter GR, Nelson KG, Copper RL. The Influence of Previous Low Birth Weight on Birth Weight, Gestational Age, and Anthropometric Measurements in the Current Pregnancy. Obstetrics and Gynecology 1992; 79: 276-9.

13. Emanuel I. Invited commentary: an assessment of maternal intergenerational factors in pregnancy outcome. American Journal of Epidemiology 1997; 146: 820-5.

14. Emanuel I, Leisenring W, Williams MA, et al. The Washington State Intergenerational Study of Birth Outcomes: methodology and some comparisons of maternal birthweight and infant birthweight and gestation in four ethnic groups. Paediatric and Perinatal Epidemiology 1999; 13: 352-69.

15. Farina A, Dini B, Mattioli M, Rosa S, Rizzo N. Offspring birth weight in second-generation 'small for gestational age' infants. Prenatal Diagnosis 2010; 30: 551-4.

16. Dempsey JC, Williams MA, Luthy DA, Emanuel I, Shy K. Weight at birth and subsequent risk of preeclampsia as an adult. American Journal of Obstetrics and Gynecology 2003; 189: 494-500.

17. Rogvi R á, Forman JL, Damm P, Greisen G. Women Born Preterm or with Inappropriate Weight for Gestational Age Are at Risk of Subsequent Gestational Diabetes and PreEclampsia. PLoS One 2012; 7: e34001.

18. World Health Organisation. Maternal anthropometry and pregnancy outcomes: a WHO collaborative study. Bulletin of the World Health Organization 1995; 73 (Supp): 1-98.

19. Ceesay SM, Prentice AM, Cole TJ, et al. Effect on birth weight and perinatal mortality of dietary supplements in rural Gambia: 5 year randomized controlled trial. British Medical Journal 1997; 315: 786-90.

\title{
Tuberculous meningitis in adults: a prospective study at a tertiary referral centre in Sri Lanka
}

\section{S A C U Gunawardhana, S C Somaratne, M A H Fernando, P S Gunaratne}

(Index words: tuberculous meningitis, clinical presentation, magnetic resonance imaging, Sri Lanka)

\begin{abstract}
Introduction Central nervous system tuberculosis is the most severe form of extrapulmonary TB and it is associated with a substantial morbidity and mortality.

Objectives To describe the demographic profile, clinical features, laboratory and imaging results of a cohort of adult patients with TBM (Tuberculous meningitis).

Methods This study encompasses a prospective analysis of all adult cases of TBM diagnosed from 1st January 2010 to 31st December 2011 in the Neurology unit 2, National Hospital of Sri Lanka. Consensus case defini-
\end{abstract}

tions for TBM were used for clinical case classification and patients were given a definite, probable, or possible tuberculous meningitis status accordingly.

Results A total of 89 patients fulfilled the established diagnostic criteria for TBM and there were 22 definitive cases, 46 probable cases and 21 possible cases. The mean age of the series was 44 years and $56(63 \%)$ were males. TBM presented with fever in $64(71 \%)$, general constitutional symptoms in $61(68 \%)$, headache in 53 (59\%), and diminished level of consciousness in 36 (40\%) patients. CSF biochemistry revealed elevated protein in all patients. MRI brain showed meningeal

Unit 2, Institute of Neurology, National Hospital of Sri Lanka.

Correspondence: SACUG, e-mail: <champikagu@yahoo.com>. Received 10 July and revised version accepted 11 October 2012. Competing interests: none declared. 
enhancement in 73 (82\%). Twenty four (27\%) died during hospitalisation and out of 65 who survived 44 (49\%) had residual sequelae at the time of discharge.

Conclusions MRI evidence and biochemical analysis of CSF are still the main supportive diagnostic modalities. TBM is a relatively common but difficult to diagnose disease, which results in significant morbidity and mortality.

Ceylon Medical Journal 2013; 58: 21-25

\section{Introduction}

Tuberculosis remains a global epidemic especially in developing countries. In 2010, 9 million new cases and 1.5 million deaths related to tuberculosis were reported and of them $50 \%$ were tuberculous meningitis (TBM) [1].

Many studies have led to the frequent generalisation that $5-15 \%$ of individuals exposed to tuberculous bacilli will develop symptomatic disease [2]. Central nervous system (CNS) tuberculosis occurs in approximately $1 \%$ of all patients with symptomatic tuberculosis [2]. CNS tuberculosis (TB) could occur in the form of TB meningitis, milliary TB, parenchymal TB, tuberculomas or TB myelitis. In patients with TBM, neurological sequelae are common, and the case fatality rate has been estimated to vary from $15 \%$ to $60 \%$ [2].

Central nervous system tuberculos is the most severe form of extrapulmonary TB and it is associated with substantial morbidity and mortality. The outcome of this disease is especially grave in the developing countries due to lack of resources for early diagnosis and poor awareness of the disease among general public [3].

In many instances, the diagnosis and there by the treatment of CNS tuberculosis is delayed owing to poor understanding of the disease pathogenesis and unavailability of rapid, sensitive, and affordable diagnostic tests. The cornerstone of early diagnosis and initiation of appropriate treatment are solely based on higher degree of clinical suspicion and are mandatory for better outcome of this grave condition. Hence, it is important to identify and understand the clinical pattern and disease spectrum of TBM to arrive at an early diagnosis. The availability of data related to TBM from Sri Lanka is limited. Hence, the objective of this study was to describe the demographic profile, clinical features, laboratory and imaging results of a cohort of adult patients with TBM.

\section{Methods}

The study population consisted of patients admitted to the largest tertiary care teaching hospital in Colombo, Sri Lanka. This study is a prospective analysis of all adult (age $>12$ years) cases of TBM diagnosed from 1st January 2010 to 31 st December 2011 in the neurology unit 2, National Hospital of Sri Lanka. Consensus case definitions for TBM were used for clinical case classification [4].
Patients were given a definite, probable, or possible tuberculous meningitis status depending on clinical, laboratory, and radiological findings.

\section{Consensus case definitions}

Clinical entry criteria - Symptoms and signs of meningitis including one or more of the following: headache, irritability, vomiting, fever, neck stiffness, convulsions, focal neurological deficits, altered consciousness, or lethargy. Table 1 shows the diagnostic criteria used for classification of definite, probable, and possible TBM.

Table 1. Diagnostic criteria for classification of definite, probable, and possible tuberculous meningitis

\begin{tabular}{|c|c|}
\hline & Diagnostic score \\
\hline Clinical criteria & $\begin{array}{l}\text { Maximum category } \\
\text { score }=6\end{array}$ \\
\hline Duration more than 5 days & 4 \\
\hline Constitutional symptoms & 2 \\
\hline $\begin{array}{l}\text { Recent close contact of } \mathrm{TB} \text { or } \\
\text { positive Monteux test }\end{array}$ & 2 \\
\hline Focal neurological signs & 1 \\
\hline Cranial nerve palsies & 1 \\
\hline Altered consciousness & 1 \\
\hline CSF Criteria & $\begin{array}{l}\text { Maximum category } \\
\text { score }=4\end{array}$ \\
\hline Appearance - clear & 1 \\
\hline Cells $10-500 / \mu 1$ per $\mu 1$ & 1 \\
\hline Lymphocyte predominance $>50 \%$ & 1 \\
\hline Protein $>100 \mathrm{mgd} / \mathrm{dl}$ & 1 \\
\hline Sugar $<50 \%$ of plasma sugar & 1 \\
\hline Cerebral imaging criteria & $\begin{array}{l}\text { Maximum category } \\
\text { score }=6\end{array}$ \\
\hline Hydrocephalus & 1 \\
\hline Basal meningeal enhancement & 2 \\
\hline Infarction & 1 \\
\hline Tuberculoma & 2 \\
\hline $\begin{array}{l}\text { Evidence of tuberculosis } \\
\text { in other sites }\end{array}$ & $\begin{array}{l}\text { Maximum category } \\
\text { score }=4\end{array}$ \\
\hline $\begin{array}{l}\text { Chest Xray evidence of active } \\
\text { pulmonary TB }\end{array}$ & 2 \\
\hline $\begin{array}{l}\text { Positive AFB or TB PCR in } \\
\text { extra CNS sites }\end{array}$ & 2 \\
\hline
\end{tabular}




\section{Definite tuberculous meningitis}

Patients should fulfill criterion A or B:

A. Clinical entry criteria plus one or more of the following: acid-fast bacilli seen in the cerebro-spinal fluid (CSF), Mycobacterium tuberculosis cultured from the CSF or a CSF positive polymerase chain reaction test for TB (TB PCR).

B. Acid-fast bacilli seen in the context of histological changes consistent with tuberculosis in the brain or spinal cord with suggestive symptoms or signs and CSF changes or visible meningitis (on autopsy).

\section{Probable tuberculous meningitis}

Clinical entry criteria plus a total diagnostic score of 10 or more points (when cerebral imaging is not available) or 12 or more points (when cerebral imaging is available) plus exclusion of alternative diagnoses. At least 2 points should come either from CSF or cerebral imaging criteria.

\section{Possible tuberculous meningitis}

Clinical entry criteria plus a total diagnostic score of 6-9 points (when cerebral imaging is not available) or 6-11 points (when cerebral imaging is available) plus exclusion of alternative diagnoses.

Patients with tuberculoma without evidence of meningeal involvement, tuberculous myelitis and spinal tuberculosis were excluded. In patients with repeated admissions, only the first hospital admission was reviewed. Patients were classified into three clinical stages according to clinical severity at admission using the British Medical Research Council TBM grades.

Stage I (Early): Nonspecific symptoms and signs with no clouding of consciousness or neurological deficits.

Stage II (Intermediate): Lethargy or alteration of behaviour, meningeal irritation or minor neurological deficits.

Stage III (Advanced): Abnormal movements, convulsions, and stupor or coma.

Variables related to demographic characteristics, clinical features, laboratory, bacteriological and radiological investigations, modality of treatment and disease outcome were analysed. Data entry and analysis were carried out using SPSS software (Release 17.0).

\section{Results}

A total of 121 cases were treated as TBM over the period of 2 years. Out of these, 89 fulfilled the established diagnostic criteria for diagnosis of definitive, probable or possible TBM and 32 were excluded from the analysis. There were 22 definitive cases, 4 probable cases and 21 possible BM cases. Their clinical features along with laboratory and radiological data are summarized in Table 2.
Table 2. Frequency of clinical, laboratory and radiological features

\begin{tabular}{|c|c|c|}
\hline Clinical features & Frequency & $\%$ \\
\hline Headache & 53 & 59 \\
\hline Fever & 64 & 71 \\
\hline Constitutional symptoms & 61 & 68 \\
\hline Seizures & 12 & 13 \\
\hline Altered consciousness & 36 & 40 \\
\hline Meningeal irritation & 44 & 47 \\
\hline Cranial nerve palsies & 23 & 26 \\
\hline Papilledema & 7 & 8 \\
\hline Hemiparesis & 7 & 8 \\
\hline \multicolumn{3}{|l|}{ CSF findings } \\
\hline Protein $>50 \mathrm{~g} / \mathrm{dl}$ & 89 & 100 \\
\hline \multicolumn{3}{|l|}{ Cells } \\
\hline Lymphocytes $>50 \%$ & 66 & 74 \\
\hline Polymorphs $>50 \%$ & 17 & 19 \\
\hline Sugar $<50 \%$ of plasma sugar & 54 & 57 \\
\hline $\begin{array}{l}\text { AFB in CSF } \\
\text { (Criteria for definitive diagnosis) }\end{array}$ & 0 & \\
\hline $\begin{array}{l}\text { TB PCR positivity } \\
\text { (Criteria for definitive diagnosis) }\end{array}$ & $21 / 68$ & 29 \\
\hline $\begin{array}{l}\text { CSF culture positive for TB } \\
\text { (Criteria for definitive diagnosis) }\end{array}$ & $11 / 73$ & 21 \\
\hline \multicolumn{3}{|l|}{ Radiological findings } \\
\hline \multicolumn{3}{|l|}{ Contrast CT brain } \\
\hline \multicolumn{3}{|l|}{ MRI brain with contrast } \\
\hline Tuberculoma & 21 & 23 \\
\hline Meningeal enhancement & 73 & 82 \\
\hline Infarction & 15 & 17 \\
\hline Hydrocephalus & 16 & 18 \\
\hline
\end{tabular}

The mean age of the series was $44 \pm 13.5$ years and the age ranged from 12 years to 82 years with the median of 36 years. Fifty six $(63 \%)$ were males. TBM presented with fever in $64(71 \%)$, general constitutional symptoms in $61(68 \%)$, headache in $53(59 \%)$, and diminished level of consciou-sness in $36(40 \%)$ patients. On physical examination nuchal rigidity was present in $16(18 \%)$, coma in $36(40 \%)$ and cranial nerve palsies in $23(26 \%)$ patients. Sixth nerve palsy was the commonest followed by seventh nerve and bulbar palsies.

On admission, $24(27 \%)$ were in stage I, $36(40 \%)$ were in stage II, and $29(32 \%)$ were in stage III of the disease. Eleven patients $(12 \%)$ had history of pulmonary TB more than three years before the diagnosis of TBM was made. All had completed the course of antituberculous medication for pulmonary tuberculosis. 
Human immune deficiency virus (HIV) was present only in one patient. Early complications of TBM included syndrome of inappropriate anti diuretic hormone secretion (SIADH) in $33(37 \%)$, seizures in $12(13 \%)$ and hemiparesis in $7(8 \%)$ patients.

CSF was analysed in all patients. CSF biochemistry (median and IQR) were as follows: Glucose 24, 17-32 mg/ $\mathrm{dl}$, protein $125,68-300 \mathrm{mg} / \mathrm{dl}$ and white blood cell count 28 , $0-288$ per $\mu 1$. Mononuclear cells predominated in 66 patients $(74 \%)$. CSF microbiological analysis revealed negative acid-fast stain in all patients, positive acid-fast bacillus (AFB) culture in $11 / 73$ patients $(21 \%)$ and positive polymerase chain reaction of TB PCR in 21 out of $68(31 \%)$ patients.

CT brain was performed in 57 patients (64\%). It was normal in $28(50 \%)$ There was hydrocephalus in $12(13 \%)$, ischaemic lesions in $15(17 \%)$ and other abnormalities in five $(6 \%)$. Meningeal enhancement was not detected in any of the contrast images. MRI brain was performed in all patients. The study was normal in $13(15 \%)$ and showed hydrocephalus in $16(18 \%)$, meningeal enhancement in 73 (82\%), enhancing lesions in 14 (16\%) and ischaemic lesions in $15(17 \%)$. Chest radiographs were normal in 83 cases $(88 \%)$ and changes suggestive of old TB were detected in $6(8 \%)$.

Anti-tuberculous treatment consisted of five drugs (rifampicin, isoniazid, pyrazinamide, ethambutol and streptomycin) in 62 patients $(76 \%)$ and four drugs (without streptomycin) in 17 (19\%). Other medications included prednislone in all patients and anticonvulsants in eleven (12\%). Three (3\%) underwent ventriculo-peritoneal shunt for hydrocephalus. Twenty four $(27 \%)$ died during hospitalisation and out of 65 who survived 44 (49\%) had residual sequelae at the time of leaving hospital whereas $21(23 \%)$ patients recovered completely.

\section{Discussion}

TBM is a common infectious disease with a grave outcome especially in low income countries like Sri Lanka. In comparison to the other series from the region, the number of cases for the given period in the reported series is higher $[3,6,7]$. This could be explained by the fact that National Hospital is the largest tertiary referral center in Sri Lanka. According to the consensus diagnostic criteria, majority of patients belonged to 'probable' or 'possible' diagnosis group. This is in keeping with most of the other reported series [2-6]. Low prevalence of definitive cases signifies difficulty in arriving at the diagnosis and the need for high degree of suspicion for early diagnosis of TBM.

Mean age of the series was 44 years. The series did not include paediatric cases. It was rare in the elderly. In contrast to some of the Asian studies, majority had TBM as the first presentation of TB. Only $12 \%$ had suffered pulmonary or pleural disease previously. Surprisingly percentage of HIV-infected patients was low in the study. This may reflect the relatively low prevalence of HIV infection in Sri Lanka.

As in many reported series headache, fever and constitutional symptoms were common presenting symptoms while nuchal rigidity was absent in most cases [3-9]. Twenty six percent of TBM presented with diminished level of consciousness. Remarkably higher prevalence of impaired consciousness with low incidence of nuchal rigidity in the reported series highlight the difficulties of recognising presentations that TBM may adopt. As in other reports, imaging of brain, particularly MRI was useful in majority of patients [3-9]. CT scan findings were not very promising in visualising leptomeningeal enhancement whereas MRI scans were positive for meningeal enhancement in $82 \%$ of patients. Introduction of MRI facility has changed the diagnostic challenges related to TBM and it also has improved the specificity of the diagnosis according to the new consensus criteria. Therefore the authors recommend MRI brain as an essential investigation for any complicated meningitis.

CSF analysis was similar to those of other studies, with moderately decreased glucose, moderate to highly increased protein and mild to moderately increased white blood cell count with predominant lymphocytes. Mononuclear cells predominated in the CSF of $>50 \%$ of patients. CSF protein was essentially increased in all patients. In contrast to most of the other studies, microbiological evidence of tuberculosis in CSF was negative in the majority of reported series [4-7]. This may be due to the microbiological techniques employed including the quantity of CSF and sample transport methods.

In most patients the diagnosis of TBM was suspected on the basis of the clinical picture, biochemical results of $\mathrm{CSF}$ analysis, and neuro imaging evidence. Acid-fast stain in CSF was negative in all patients of this study. CSF TB culture and TB PCR were positive in a minority of patients.

Outcome was poor in our study, with a mortality of $27 \%$ and persistence of sequelae in $44 \%$ of patients. Most other published studies give a better prognosis for TBM [3-10]. Being a tertiary referral centre receiving complicated patients and complexity of the clinical condition leading to delayed diagnosis are potential explanations for this situation. Due to the characteristics of the present study, a relatively small number of cases, and the absence of long term follow up, authors may have failed to describe some important factors related to TBM. There is still room for further work related to this area.

In conclusion, clinical presentation of TBM is complex in many occasions and definitive microbiological evidence has limited value in diagnosis of the disease. High degree of clinical suspicion, MRI evidence and biochemical analysis of CSF are the main supportive diagnostic modalities. TBM is difficult to diagnose but results in significant morbidity and mortality. 


\section{References}

1. World Health Organization. Global tuberculosis controlsurveillance, planning, financing. WHO report 2010. Available at: http:// www.who.int/tb/publications/ global_report/en

2. Thwaites GAH, Fisher MBI, Hemingway CCJ, Scott GDK, Solomon TEL, Innes JFGM. British Infection Society guidelines for the diagnosis and treatment of tuberculosis of the central nervous system in adults and children. Journal of Infection 2009; 59: 167-87.

3. Qureshi HU, Merwat SN, Nawaz SA, et al. Predictors of inpatient mortality in 190 adult patients with tuberculous meningitis. Journal of Pakistan Medical Association 2002; 52: 159-63.

4. Marais S, Thwaites G, Schoeman JF, et al. Tuberculous meningitis: a uniform case definition for use in clinical research. Lancet Infectious Diseases 2010; 11: 803-12.

5. Po-Chang Hsu, Chien-Chang Yang, Jung-Jr Ye, Po-Yen Huang, Ping-Cherng Chiang, Ming-Hsun Lee. Prognostic Factors of Tuberculous Meningitis in Adults: A 6-Year
Retrospective Study at a Tertiary Hospital in Northern Taiwan. Journal of Microbiology, Immunology and Infection 2010; 43: 111-8.

6. Roca B, Tornador N, Tornador E. Presentation and outcome of tuberculous meningitis in adults in province of Castellon, Spain: A retrospective study. Epidemiology and Infections 2008; 136: 1455-62.

7. Phypers M, Harris T, Power C. CNS tuberculosis: a longitudinal analysis of epidemiological and clinical features. International Journal of Tuberculosis and Lung Disease 2006; 10: 99-101.

8. Thwaites GE, Duc BN, Dung NH, et al. The influence of HIV infection on clinical presentation, response to treatment, and outcome in adults with Tuberculous meningitis. Journal of Infectious Diseases 2005; 192: 2134-41.

9. Bhigjee AI, Padayachee R, Paruk H, Hallwirth-Pillay KD, Marais S, Connoly C. Diagnosis of tuberculous meningitis: clinical and laboratoryparameters. International Journal of Infectious Diseases 2007; 11: 348-54.

10. Lu CH, Chang WN, Chang HW. The prognostic factors of adult tuberculous meningitis. Infection 2001; 29: 299-304. 